\title{
MUSIC for sub/millimeter astrophysics
}

\author{
Philip R. Maloney ${ }^{a}$, Nicole G. Czakon ${ }^{b}$, Peter K. Day ${ }^{c}$, Thomas P. Downes ${ }^{b}$, Ran Duan ${ }^{b}$, \\ Jiansong Gao ${ }^{d}$, Jason Glenn ${ }^{a}$, Sunil R. Golwala ${ }^{b}$, Matt I. Hollister ${ }^{b}$, Henry G. LeDuc ${ }^{c}$, \\ Benjamin A. Mazin ${ }^{e}$, Sean G. McHugh ${ }^{e}$, Omid Noroozian ${ }^{b}$, Hien T. Nguyen ${ }^{c}$, Jack Sayers ${ }^{c}$, \\ James A. Schlaerth ${ }^{a}$, Seth Siegel ${ }^{b}$, John E. Vaillancourt ${ }^{f}$, Anastasios Vayonakis ${ }^{b}$, Philip \\ Wilson $^{c}$, Jonas Zmuidzinas ${ }^{b}$ \\ ${ }^{a}$ Center for Astrophysics and Space Astronomy, University of Colorado, Boulder, CO \\ ${ }^{b}$ California Institute of Technology, Pasadena, CA \\ ${ }^{c}$ Jet Propulsion Laboratory, Pasadena, CA \\ ${ }^{d}$ National Institute of Standards and Technology, Boulder, CO \\ eUniv. of California, Santa Barbara, CA

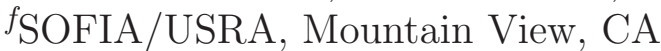

\begin{abstract}
MUSIC (the Multiwavelength Submillimeter kinetic Inductance Camera) is an instrument being developed for the Caltech Submillimeter Observatory by Caltech, JPL, the University of Colorado, and UCSB. MUSIC uses microwave kinetic inductance detectors (MKIDs) - superconducting micro-resonators - as photon detectors. The readout is almost entirely at room temperature and is highly multiplexed. MUSIC will have 576 spatial pixels in four bands at 850, 1100, 1300 and 2000 microns. MUSIC is scheduled for deployment at the CSO in the winter of 2010/2011. We present an overview of the camera design and readout and describe the current status of the instrument and some results from the highly successful May/June 2010 observing run at the CSO with the prototype camera, which verified the performance of the complete system (optics, antennas/filters, resonators, and readout) and produced the first simultaneous 3-color observations with any MKID camera.
\end{abstract}

Keywords: Superconducting submillimeter and millimeter wave detectors, radio telescopes and instrumentation

\section{INTRODUCTION}

A unique window onto the epoch of galaxy and supermassive black hole formation is provided by observations at millimeter and submillimeter wavelengths; due to the negative K-correction produced by redshifting, these wavelengths are ideally matched to the spectral energy distributions of high-redshift, dusty objects. Observations to date have shown that galaxy formation peaked at a redshift $z \sim 2$ and identified a major population of objects referred to as submillimeter galaxies ${ }^{1}$ (SMGs), so named because they radiate the overwhelming majority of their luminosity at far-infrared to millimeter wavelengths via thermal dust emission. Further progress in obtaining an accurate picture of the formation and evolution of dusty, gas-rich galaxies will require new, large-scale surveys at submillimeter/millimeter wavelengths.

The development of large-format arrays of incoherent detectors for sub/millimeter astronomy has proven to be technologically challenging, especially due to the difficulties in reading out thousands of detectors simultaneously. Considerable progress has been made recently using a novel type of device, microwave kinetic inductors, or MKIDS. ${ }^{3,12,17}$ We are developing a 576-pixel, 4-color astronomical instrument, MUSIC, which will be deployed at the Caltech Submillimeter Observatory on Mauna Kea in the winter of 2010/2011.

For further information contact Philip Maloney: philip.maloney@colorado.edu or 3034925055 


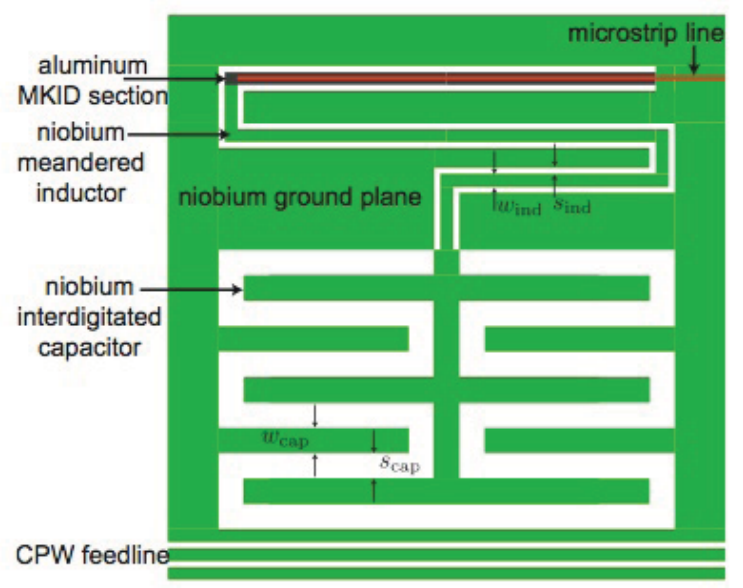

Figure 1. Schematic of an MKID resonator design with an interdigitated capacitor. The resonator is fed via microstrip from a planar multi-slot antenna to the absorbing, aluminum portion of the resonator; the remainder of the circuit is made of niobium, which does not absorb below $700 \mathrm{GHz}$. The IDC portion is capacitively coupled to a CPW feedline; the distance to the feedline determines the coupling $Q, Q_{c}$.

\section{MKID DETECTORS}

The physics of MKIDs and their applications are discussed in detail by Mazin, ${ }^{11}$ and references therein; here we present only the directly relevant aspects.

Superconductors have a non-zero impedance for ac currents, as the paired-up electrons (known as Cooper pairs) which carry the supercurrent can be accelerated and decelerated by an electric field, allowing energy to be stored in (or extracted from) the kinetic energy of the Cooper pairs. This effect of the inertia of the Cooper pairs contributes what is known as the kinetic inductance.

At finite temperatures below the superconducting transition temperature $T_{c}$, a small fraction of the electrons are thermally excited out of the Cooper pair states. These "quasiparticles" produce the small surface resistance $R_{s}$. The net result is that the superconductor has a surface impedance to ac currents $Z_{s}=R_{s}+i \omega L_{s}$ that is almost purely inductive, $\omega L_{s} \gg R_{s}$. The Cooper pairs are bound together by the electron-phonon interaction with an energy $2 \Delta \approx 3.5 k T_{c}$. Photons with energies $>2 \Delta$ that are absorbed by the superconductor can break apart Cooper pairs, resulting in an increase in the quasiparticle density and therefore a change in the surface impedance $Z_{s}$. By measuring this change in the impedance, which alters both the resonant frequency $f$ and the resonator quality factor $Q$, the optical load on the superconductor can be derived. For aluminum, the binding energy corresponds to a minimum photon frequency of $90 \mathrm{GHz}$.

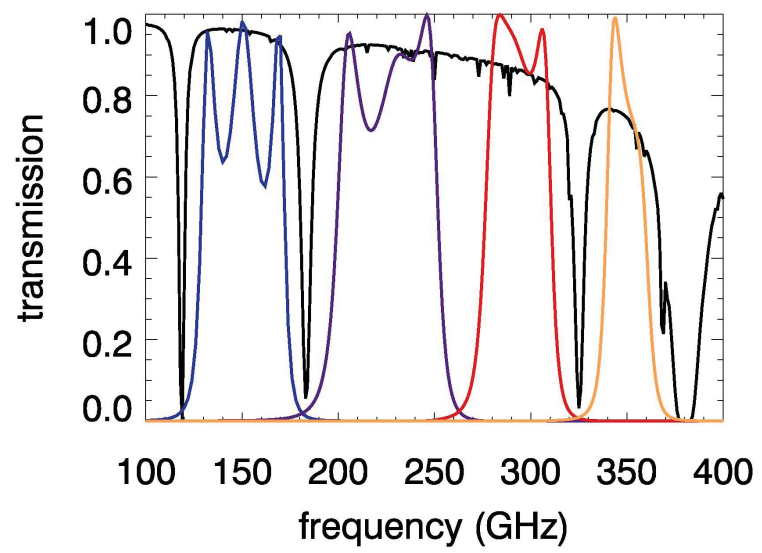

Figure 2. MUSIC filter bands (color) overlain on the atmospheric transmission for $1.5 \mathrm{~mm}$ pwv.

To implement this as a photon detector, we use thin superconducting films of aluminum and niobium as part of a lithographed resonator circuit (Figure 1). The original resonator designs used simple CPW geometry; 


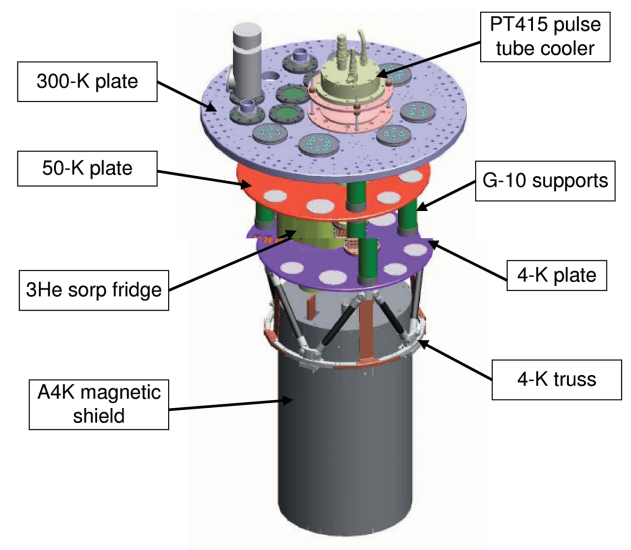

Figure 3. Illustration of the interior of the MUSIC cryostat, showing the 3 stages $(300 \mathrm{~K}, 50 \mathrm{~K}$, and $4 \mathrm{~K})$, the top of the pulse-tube cooler and ${ }^{3} \mathrm{He}$ fridge, the G-10 and truss supports for the $4 \mathrm{~K}$ plate, and the magnetic shield. Hollister et al. ${ }^{9}$ provide a detailed discussion of the cryomechanical design of the cryostat.

this has been modified to use interdigitated capacitor sections (IDCs) ${ }^{13}$ to reduce two-level system noise, ${ }^{6-8}$ with stepped-impedance filters to cut down on direct radiation pick-up by the IDCs. ${ }^{16}$ The hybrid quarter-wave resonators have Lorentzian profiles (with unit transmission well away from resonance) and are designed to have intrinsically high Qs $\left(>10^{4}\right)$. For a resonator frequency $f \sim 3 \mathrm{GHz}$, the FWHM is only $\sim 100 \mathrm{kHz}$; hence it is possible to read out many resonators simultaneously in a few hundred $\mathrm{MHz}$ bandwidth.

For the MUSIC design, the resonators are antenna-coupled, fed radiation via microstrip from broad-band, lithographic planar multi-slot antennas; ${ }^{4}$ this allows us to form the antenna beams, and also to define the bandpasses via on-chip filtering. Traditionally, the passbands of sub/mm instruments have been defined by using metal-mesh filters in combination with infrared-blocking materials such as teflon or fluorogold to make quasioptical filters. However, these designs make multicolor pixels impossible, since the entire array is illuminated by the same frequency band. A great increase in throughput can be achieved by allowing a single spatial pixel to feed multiple detectors at different frequencies. This is achieved in MUSIC by using superconducting, on-chip, lumped-element bandpass filters, ${ }^{10}$ which are placed in-line with the microstrip connecting the antenna and the detector. The MUSIC filter bands are shown in Figure 2, along with the atmospheric transmission.

Each pixel consists of an antenna, an in-phase combining microstrip feed network, the on-chip filters for bandpass definition, and four resonators, one for each band. MUSIC will have 8 tiles of $6 \times 12$ spatial pixels in four colors $(150,230,290,350 \mathrm{GHz}=2.0 \mathrm{~mm}, 1.3 \mathrm{~mm}, 1.1 \mathrm{~mm}, 850 \mu \mathrm{m})$ read out in a $\sim 550 \mathrm{MHz}$ bandpass, for a total of 2304 channels (288 MKIDs/tile $\times 8$ tiles). Two tiles are read out with a single HEMT amplifier, so a total of 8 LNAs are needed, and only 16 coaxes are required for readout. In addition to the advantages in throughput and efficient use of the focal plane, we can use the bandpass-defined responses for each antenna to do multicolor sky subtraction.

\section{CRYOSTAT AND CAMERA DESIGN}

The cryostat for MUSIC was manufactured by High Precision Devices in Boulder, CO and was delivered on schedule in September of 2008. It has been thoroughly tested thermally and mechanically, and has been modified to accomodate the cold focal plane hardware. MUSIC will use a Cryomech PT-415 pulse-tube to cool the first and second stages of the instrument to 50 and $4 \mathrm{~K}$, respectively. Final cooling to the $T \sim 250 \mathrm{mK}$ required by the MKID focal plane module is obtained from a Chase Cryogenics He-10 two-stage ${ }^{3}$ He adsorption refrigerator.

Details of the cryomechanical design (see Figure 3) are presented in Hollister et al. ${ }^{9}$ Because MKIDs, like other superconducting devices, are sensitive to magnetic fields, a magnetic shield, manufactured by Amuneal Manufacturing Corporation, will enclose the focal plane. Testing in both the lab and at the CSO with the prototype camera (see $§ 5$ ) confirm that the shield design reduces the MKID response to negligible levels.

MUSIC is intended for operations at the CSO, with the primary goal being large-scale surveys for submillimeter galaxies. Hence maximizing the mapping speed (i.e, the sensitivity per pixel given a fixed number of detectors) is achieved for pixel spacings of $\simeq 2(\mathrm{f} / \#) \lambda$. This mandates a larger field of view than is provided by 
the 8 arcmin FOV of the existing CSO relay optics; we are therefore designing and fabricating new relay optics, which will be installed at the CSO during summer shutdown of 2010 .

Details of the optical design of both the camera and the new relay optics are presented by Sayers et al. ${ }^{14}$ MUSIC will have a $14^{\prime} \mathrm{FOV}$, and PSFs of 22, 25, 31, and 45 arcseconds at 350, 290, 230, and $150 \mathrm{GHz}$, respectively; the predicted mapping speed for high-redshift, dusty objects is $\sim 600 \operatorname{arcmin}^{2} \mathrm{mJy}^{-2} \mathrm{hr}^{-1}$ (scaled to $1.1 \mathrm{~mm}$ ). Note that this prediction takes into account both realistic sky noise (based on measurements with the Bolocam mm-wave array ${ }^{15}$ ) and HEMT amplifier and MKID detector noise; if all of these noise contributions could be removed down to the BLIP limit, the mapping speed would be $\sim 2500 \operatorname{arcmin}^{2} \mathrm{mJy}^{-2} \mathrm{hr}^{-1}$.

The MUSIC cryostat has a large (300 mm diameter) window; the optical power through this window produces a noticeable load on the first $(50 \mathrm{~K})$ stage of the cryostat, and exceeds the cooling capacity of the second stage of the PT-415 and of the He-10 fridge. Most of this power is due to radiation that lies shortward of our waveband of interest. We therefore use a filtering scheme that involves a combination of dielectrics (HDPE) and metal-mesh filters to reduce the optical loading to negligible levels. ${ }^{9,14}$ Effective heat-sinking of the individual tiles is also crucial to mitigate substrate heating caused by absorption of radiation; ${ }^{16}$ this is achieved by depositing gold pads onto the device groundplane, which allows the use of gold wire bonds, with high thermal conductivity, between the substrate and the device box. The thermal loading on the second stage will be dominated by the dissipation of the power of the 8 HEMT LNAs. ${ }^{9}$

\section{READOUT}

The MKID resonators are capacitively coupled to a coplanar-waveguide feedline for the microwave readout. Each resonator is tuned to a different frequency within the band, spaced far enough apart that the probability of collisions (overlap of the resonator responses at significant power) is small. A tile of resonators is read out simultaneously by sending in a comb of excitation frequencies, with a tone for each resonator. We use an IQ mixing scheme to measure the complex transmission amplitude for each resonator; as the frequency is swept over the resonance, it traces an "IQ-loop" in the complex plane (Figure 4), which contains the phase and amplitude information. The quantity of interest is the change in transmission between the probe signal and an identical signal that is not sent past the resonators*, given by (in the small-signal limit)

$$
\delta S_{21}=\frac{Q^{2}}{Q_{c}}\left(\delta \frac{1}{Q_{i}}+2 i \frac{\delta f}{f_{0}}\right)
$$

where $f_{0}$ is the unaltered resonance frequency, $Q_{i}$ is the internal quality factor of the resonator and $Q_{c}$ is the coupling $Q$ between the resonator and the feedline. The first term is the fractional change in amplitude (or $Q$ ) of the resonator, the second is the fractional frequency response.

A single pair of coaxial cables and a low-noise HEMT amplifier are the only cryogenic electronics needed. A detailed description of the readout is provided by Duan et al., this conference. ${ }^{5}$

A schematic showing the readout for one tile of MKIDs is shown in Figure 5. The multiplexed probe signal is generated at baseband frequency $\left(\nu_{b} \simeq 0-200 \mathrm{MHz}\right)$ using high-speed, large-bandwidth DACs to play back a predefined, easily modified waveform stored in memory. Easy modification of the waveform is required because the resonant frequencies of the MKIDs change as a function of observing conditions (i.e., telescope elevation angle or amount of water vapor) - this was observed with the prototype DemoCam testbed in its Spring 2007 observing run. The baseband DAC signals are then upconverted to the microwave band and sent into the cryostat to excite the resonators. By using a dual-DAC scheme with an up-converting IQ mixer, and a similar scheme with dual ADCs on the output side, the need for single-sideband conversion is avoided. The up-converted digital output must be demodulated to measure the complex transmission amplitudes $S_{21}$ of the readout tones past the resonators, and decimated to a reasonable rate for storage to disk. This is accomplished by an FPGA core (built around a CASPER ROACH board with a Xilinx SX-95T FPGA), using a two-stage FFT narrow-band channelizer ${ }^{5}$ see Figure 6.

\footnotetext{
*In other words, we use a homodyne mixing scheme.
} 


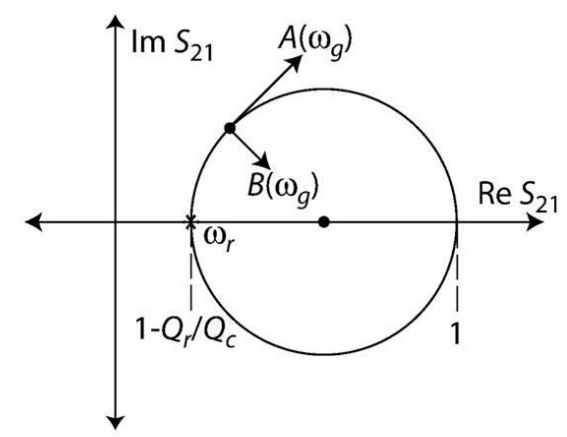

Figure 4. Resonance loop (i.e., the complex transmission $\left.S_{21}\right)$ in the complex plane. $Q_{c}$ is the coupling $Q$, while $Q_{r}$ is the sum of the coupling and internal resonator $Q_{\mathrm{s}}$, $1 / Q_{r}=1 / Q_{i}+1 / Q_{c}$. The tangent to the loop at some frequency $\omega_{g}, A\left(\omega_{g}\right)$, is the phase (frequency shift) direction, while the amplitude (dissipation) response is in the radial direction, $B\left(\omega_{g}\right)$. The resonance frequency is $\omega_{r}$; here the phase response is purely imaginary, and the amplitude response is purely real (for an ideal resonance loop). The ratio of frequency to amplitude response is typically $\sim 4$, depending on temperature; this ratio determines the "quasiparticle direction" at any point on the loop.

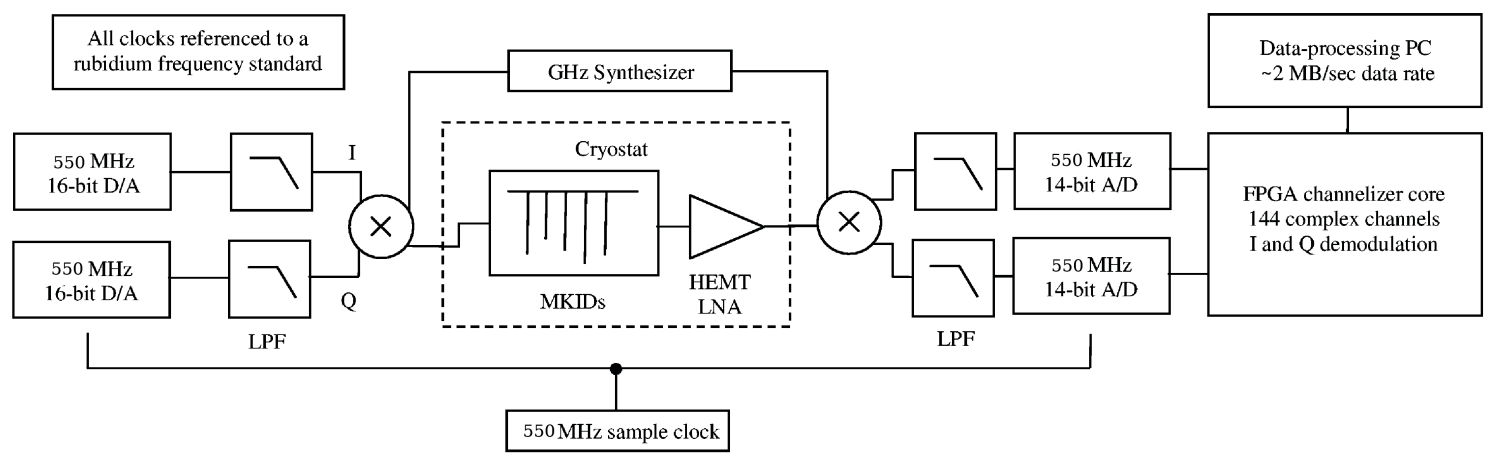

Figure 5. Readout for one tile of resonators in MUSIC: DACs generate the frequency comb which is upconverted to $\sim 3 \mathrm{GHz}$ to excite resonators, amplified and then digitized by the ADCs, then passed to the FPGA channelizer core for demodulation and decimation.

Generation of the waveform for the readout requires considerable care. With over 100 readout tones in each waveform, the instantaneous amplitude of the waveform can be very different from the sum of the carrier powers, since the phases vary; in other words, the envelope of the carrier waveform exhibits large variation. This can result in clipping - the amplitude of the carrier waveform at a particular time sample exceeds the limits of the DAC, even though the average power is within the DAC limits.

The simplest solution is to scale the waveform so that it fits within the DAC limits. However, this results in a poor peak-to-average ratio (PAR), which means that the SNR is greatly reduced from the limiting DAC performance. A second simple solution is to clip the waveform at the DAC limits. This does not affect the average power very much, and gives a better PAR than scaling the waveform, but has the very undesirable consequence of scattering power to non-carrier frequencies ${ }^{\dagger}$. If the level of clipping is substantial, the amount of power lost from the carrier can be a large (and variable) fraction of the original power.

A much better solution takes advantage of the small fraction of the total bandwidth that is actually occupied by resonators, and uses non-carrier tones to construct a pulse that does not contain any carrier frequencies. ${ }^{18}$ An iterative process is used: first a Dirac-like time pulse is constructed, power at carrier frequencies is removed from its Fourier transform, it is then scaled for the lost power and an IFFT operation performed, and finally it is convolved with a "clipping waveform", with the amplitude and sign of each clipping event (i.e., its amplitude is zero wherever the original carrier waveform lies within the limits of the DAC). This convolved pulse is then subtracted from the carrier waveform, and the process is iterated until no more clipping is seen ${ }^{\ddagger}$. We have tested

\footnotetext{
${ }^{\dagger}$ Clipping the waveform is equivalent to adding an appropriately scaled pulse at the time sample of the clip, and such a pulse has a white frequency spectrum.

${ }^{\ddagger}$ A further level of complication is introduced by the requirement that there also be no clipping at the ADC, which typically has lower SNR than the DAC.
} 
and implemented this clipping scheme for generation of the readout tone buffer and verified that it preserves an even distribution of power across the carriers, at the acceptable cost of introducing low-level noise in the non-carrier bins.

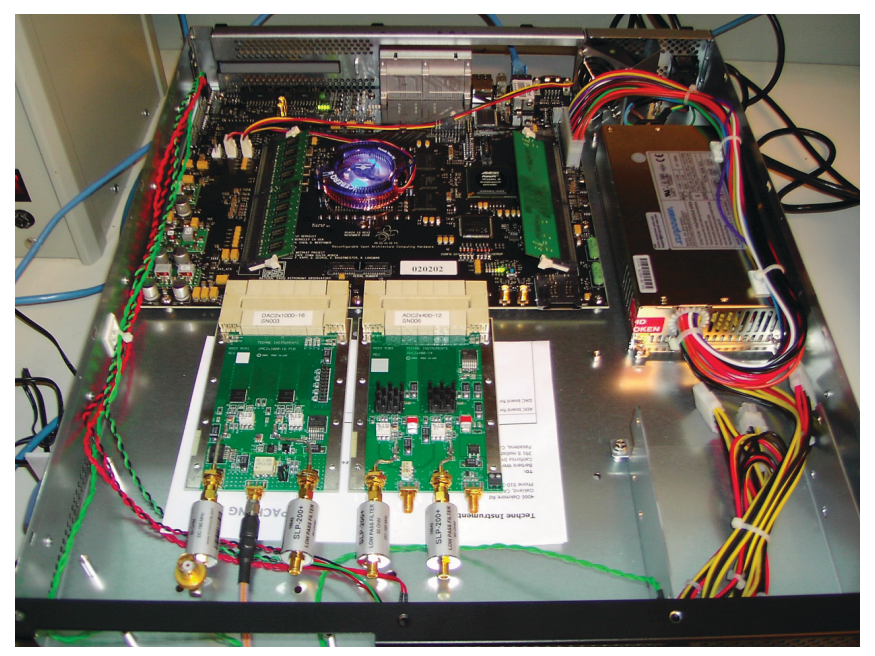

Figure 6. The readout module in its crate, as tested in May and June 2010. The DAC (left) and ADC (right) boards are at bottom, the ROACH board at the top.

\subsection{Readout system noise}

The goal for the readout is to insure that the overall noise level at the frequencies of interest $(t \sim 0.1-100$ $\mathrm{Hz}$ ) is below that of the HEMT amplifiers, which have noise temperatures $T_{n} \approx 4 \mathrm{~K}$. This mandates the use of high-resolution (16-bit for the DAC, 12-bit for the ADC) converters, but there are many other potential sources of noise within the system, such as voltage fluctuations, clock jitter, $1 / f$ noise in the DAC/ADC, etc.

Tests of the readout system comparing carrier bins (those with readout tones) to non-carrier bins demonstrated SNR ratios as expected, and satisfying the system requirements. However, within the carrier bins there are additional noise sources that significantly degrade the performance: substantial $1 / f$ noise is present below a few hundred $\mathrm{Hz}$, greatly exceeding the expected bit noise level from the ADC. This is shown by the red curve in Figure 7: plotted is the PSD of the raw $100 \mathrm{~Hz}$ output timestream, with the scale normalized to full scale of the ADC. The expected white noise level (i.e., the bit noise level of the ADC) is $-156 \mathrm{dBFS}$. At $1 \mathrm{~Hz}$, the system performance is $\sim 30 \mathrm{~dB}$ worse than the expected.

It is believed that a considerable fraction of this $1 / f$ noise is caused by fluctuations in the voltage regulator of the ADCs and DACs; this will be eliminated in the next generation of the readout by using a low-noise common voltage regulator design with a stable external reference for the DACs and ADCs. However, we also expect $1 / f$ noise from gain and phase fluctuations in the HEMT amplifiers, which are unavoidable. Therefore, in addition to identifying and eliminating sources of noise, we have also taken steps to reduce the noise by removing common-mode noise from the bins. For tone frequency separations $\Delta \nu \lesssim 10 \mathrm{MHz}$, the low-frequency noise is highly correlated, and can be successfully removed down to the white noise limit for $\nu \gtrsim 1 \mathrm{~Hz}$, as shown by the PSD of the cleaned timestream (in green) in Figure 7. To implement this for the MUSIC readout, we use an array of off-carrier tones ( $\sim 1$ for each carrier tone) to construct a noise template which is then subtracted from the carrier bins. Further work to improve the performance of the noise-subtraction algorithm, especially in the $\sim 0.1-1 \mathrm{~Hz}$ range, is in progress.

\section{MUSIC PRACTICE: DEMOCAM ENGINEERING RUN MAY/JUNE 2010}

Our testbed cryostat, used for array evaluation, readout tests, etc., has been rebuilt into a prototype for MUSIC. Originally taken to the telescope in Spring 2007 with a very early 16-pixel, 2-color array and a vastly less capable 


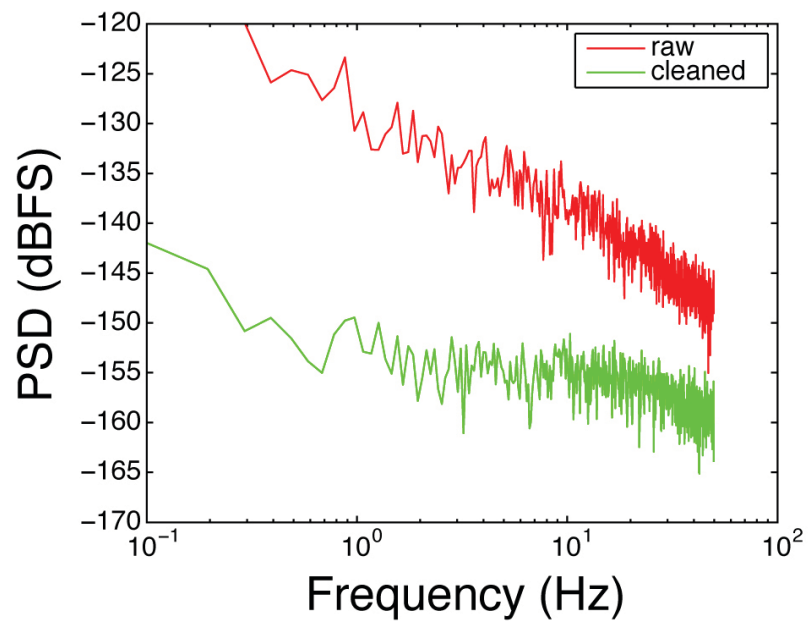

Figure 7. Power spectral density (PSD) of the audio-frequency output from the readout electronics. Red shows the PSD before cleaning, green after cleaning using a second off-carrier signal for common-mode subtraction. The expected white noise level is $-156 \mathrm{dBFS}$.

readout system, it has been completely rebuilt with a magnetic shield, new optics, and a new focal plane for the new array designs. The prototype, nicknamed DemoCam, was shipped to the CSO in early May for two observing runs, the first from the 25 th -29 th of May, the second the 19th -23 rd of June. This was the first time simultaneous multi-color readout was used.

In addition to installing the cryostat, the new optics box had to be mounted, and pre-observing tests (such as hot/cold loads) carried out to verify device and cryostat functionality, as well as mounting of the electronics and readout computer.

Because of limitations on the readout bandwidth (which will be eliminated for the final MUSIC readout), only one-half of the array was read out. In addition, the longest-wavelength band at $2 \mathrm{~mm}$ was not implemented on the array; instead, $1 / 4$ of the resonators were dark (not connected to antennas) for test and diagnostic purposes. Of the potential number of 72 resonators (18 spatial pixels with 3 observing bands, plus darks), 67 were functional, with 61 falling within the available bandpass. The final number of useful resonators was 55 (one pair were eliminated due to collision, the others were in portions of the bandpass not considered due to roll-off from lowpass filtering and the IF windowing): 14 band 1, 15 band 2, 16 band 3, and 10 dark resonators. In addition, 71 off-resonance tones for noise reduction, as discussed above, were used, for a total of 126 tones. Figure 9 shows the transmission (the magnitude of the microwave scattering parameter $S_{21}$ ) for all of the resonators present on the device taken to the telescope, while figure 8 shows the layout of the pixels on the array, color-coded by band to show the working resonators. The observing bands are separated by resonant frequency; band 2 resonators occupy the $3.0-3.13 \mathrm{GHz}$ range, band 1 the $3.13-3.23 \mathrm{GHz}$ range, and band 3 the $3.23-3.31 \mathrm{GHz}$ range, while the dark resonators lie between 3.31 and $3.4 \mathrm{GHz}$.

Because the resonator parameters $(Q$ and resonant frequency $f$ ) change as the sky loading varies (due to change in the atmospheric optical depth, or in the elevation of a source), it is necessary to use an updated set of resonance frequencies for each observation. We first upload a standard buffer of excitation tones, obtained at the summit prior to the start of the observing run, and use the readout to perform the equivalent of a network analyzer sweep (as in Figure 9), in order to find the new locations $f$ the resonances; in extreme cases, these may have shifted by $\sim 100 \mathrm{kHz}$ or more. The new buffer is then used for the observation, insuring that we will accurately determine the resonator shifts due to optical loading from the source.

All 55 resonators were read out simultaneously in four bands in $\sim 340 \mathrm{MHz}$ of bandwidth; this is the first 3 -color readout on-sky with any MKID camera. 


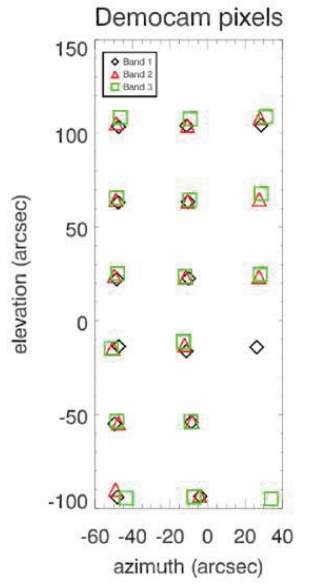

Figure 8. Spatial layout of the pixels on the resonator array used in the DemoCam May/June 2010 observing run, with functioning resonators indicated.

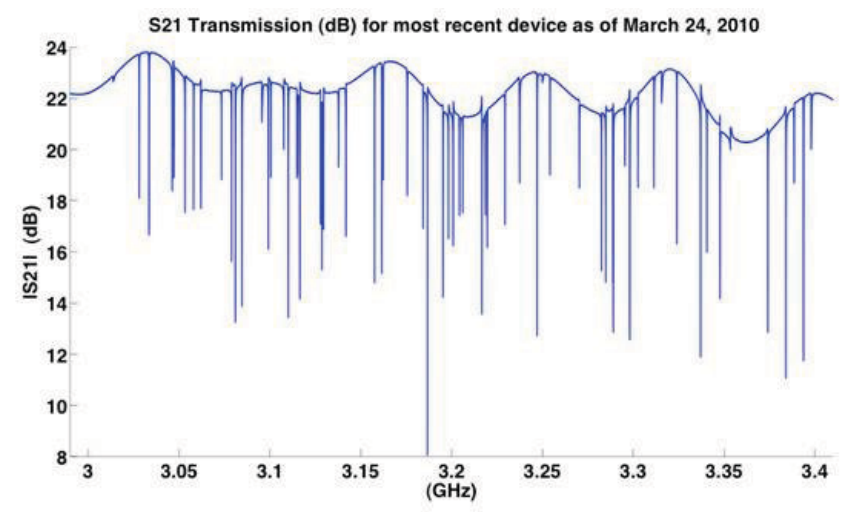

Figure 9. Network analyzer sweep showing transmission amplitudes $\left|S_{21}\right|$ for the resonators in the readout bandwidth for the device used in the DemoCam engineering run.

The data need to be processed through several steps before they are in a useful form. In general, the I and Q timstreams do not represent the desired phase and amplitude components (i.e., the resonance does not lie on the real axis, as in Figure 4), but linear combinations of the two, and the data must be projected into the correct directions. This is followed by noise removal steps, separately for the electronics noise and the sky noise. The sky noise removal algorithm is derived from that developed for Bolocam; ${ }^{15}$ for the final MUSIC pipeline this will be supplanted by a spectral sky subtraction algorithm that takes advantage of the simultaneous multicolor imaging provided by MUSIC, which should allow us to improve on the elimination of atmospheric noise.

First light on an astronomical source with the rebuilt system was obtained on the evening of Monday the 24th of May, when Saturn was (easily!) seen in the resonator timestreams. Overall, the run was extremely successful, and verified the complete end-to-end performance of the camera. The beam positions and beam sizes (which are slightly different from the final sizes, as this run used the Bolocam optics box) agreed very well with predictions, with the beam sizes within a few per cent of expected - note that the antennas and optics span more than 100 $\mathrm{GHz}$ in bandwidth. As discussed above, all of the resonators were read out simultaneously, with a new buffer (to correct for variations in loading) calculated from on-the-fly resonance fitting and uploaded at the start of each observation. The filter bandpasses are very constant from pixel to pixel and agree with predictions. The measured responsivities determined from sky dips (where the telescope zenith angle is systematically varied, thereby altering the loading as the optical depth through the atmosphere increases with airmass) agree with those derived from hot/cold loads, ${ }^{16}$ confirming that the resonators behave as anticipated. Measurements while slewing the telescope in azimuth confirmed our laboratory measurements that the magnetic shield installed in the rebuilt DemoCam has reduced the response of the MKID resonators to negligible levels; in fact, the response is so small we have not yet accurately determined it. The magnetic shield designed for the final MUSIC cryostat will produce an additional order of magnitude attenuation of exterior magnetic fields, for a total $\mathbf{B}$-field attenuation at the focal plane of $\approx 5000$.

Since this was an engineering run to test the system performance, most of the observations were of calibration sources. Figure 10 shows observations in the 3 wavebands of the ultra-compact HII region G34.3. With an extent of approximately 10 arcseconds, G34.3 is unresolved by the DemoCam beams. The images show the fractional frequency response (in other words, the frequency shift that was produced by the additional resonator loading due to the source - see equation [1]), not the flux, which is why G34.3 does not appear brighter at $850 \mu \mathrm{m}(350$ $\mathrm{GHz})$ than at $1.3 \mathrm{~mm}(230 \mathrm{GHz})$.

In Figure 11 we show extremely preliminary sensitivity estimates (note the date of the observation); these data have had an initial version of sky-noise removal, but there has been no electronic noise removal, aside from what gets eliminated by removal of the average. The data points are color-coded by band and also coded by 

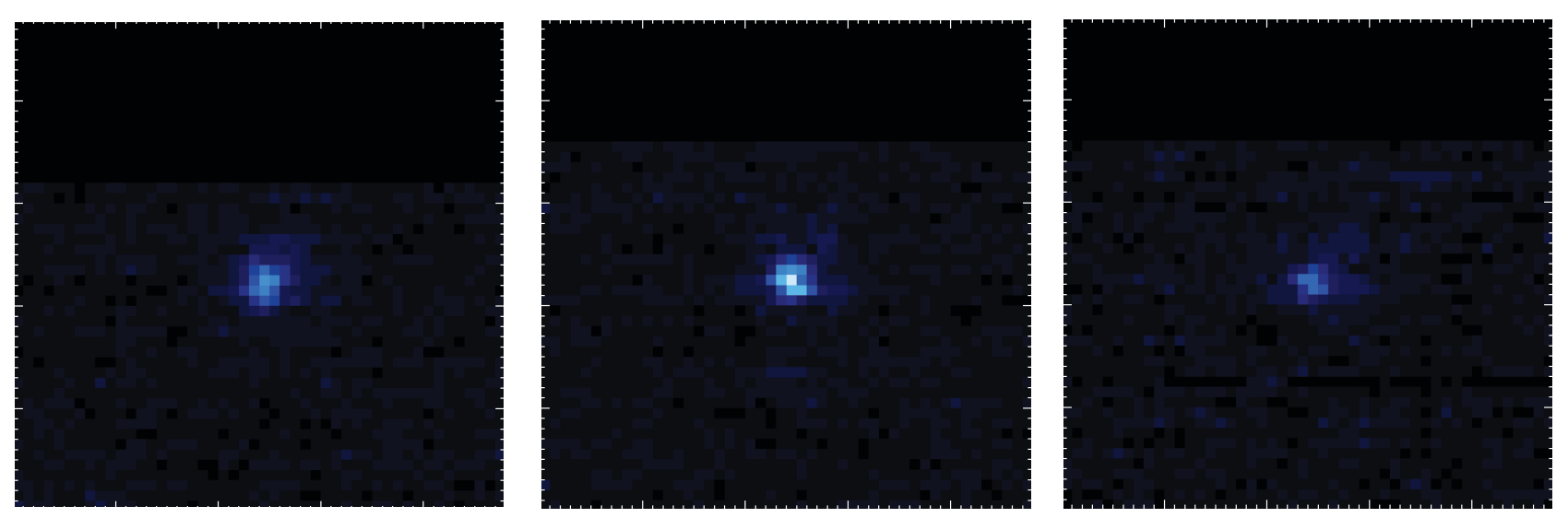

Figure 10. Observation from June 24, 2010 of the ultracompact HII region G34.3 with DemoCam. From left to right: $\lambda=1.3 \mathrm{~mm}, \lambda=1.1 \mathrm{~mm}, \lambda=850 \mu \mathrm{m}$. Brightness gives the fractional frequency response (see equation [1]), not the source flux, which is why G34.3 appears fainter at $850 \mu \mathrm{m}$ than at 1.1 or $1.3 \mathrm{~mm}$.

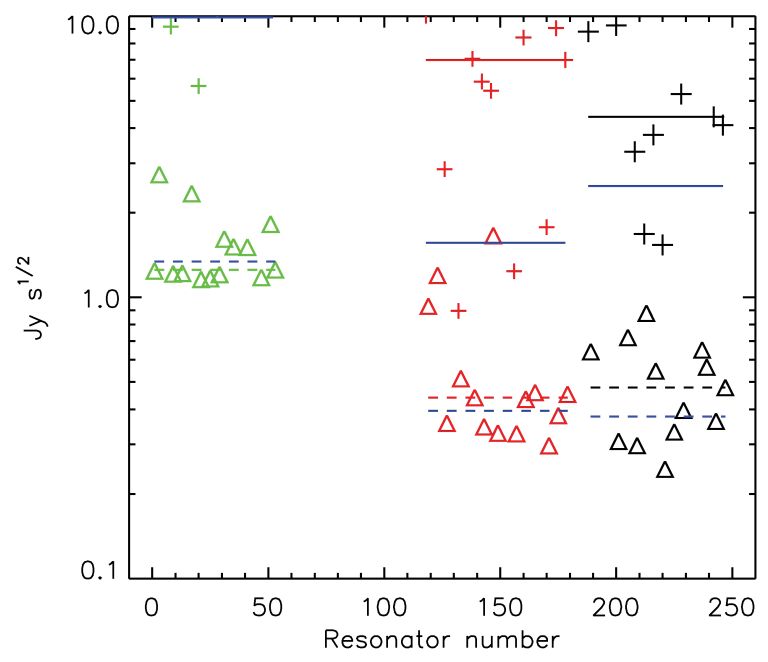

Figure 11. Very preliminary sensitivity estimate from observation of G34.3 during late June 2010 observing run with DemoCam. This observation is from June 24; other observations of this same source and of Uranus give very consistent results. Triangles are frequency (Q) responses, plus signs are amplitude (I) responses, which are inherently weaker. Data points are color-coded by band: black for $230 \mathrm{GHz}$, red for $290 \mathrm{GHz}$, green for $350 \mathrm{GHz}$. The solid and dashed lines are the median values for I and Q, respectively. The blue solid and dashed lines are the corresponding inverse-variance-weighted mean values.

symbol (triangle for frequency (Q) response, plus sign for amplitude (I) response). The black, red, and green solid and dashed lines are the median values for the I and Q responses (over all resonators) in each band, while the blue lines (the same color for all bands) are the inverse-variance-weighted means. The latter values are $\sim 400$ $\mathrm{mJy} \mathrm{s}^{1 / 2}$ for the 230 and $290 \mathrm{GHz}$ bands, and a little more than $\sim 1 \mathrm{Jy} \mathrm{s}^{1 / 2}$ for band 3 . It is very important to note that these variance-weighted means include residual sky noise and HEMT/device/readout electronics noise. The noise removal that has been applied to these data is far from optimal. In addition, it is possible to improve the signal to noise ratio by driving the resonators slightly at readout powers that are high compared to the optical loading (see the discussion in Czakon et al..$^{2}$ ) and additional laboratory work is required to establish the optimum drive power for readout. We can reasonably expect a factor of $\sim$ a few improvement in sensitivity, to levels approaching BLIP.

\section{SUMMARY}

MKIDs have a number of advantages for astronomical instrumentation. The detector fabrication is simple, requiring only $\sim 6$ levels of lithography. Since generation-recombination noise (the limiting noise level, set by phonon-electron interaction) is exponentially suppressed at temperatures well below the transition temperature, where the MKIDs are operated, $T_{c}$ uniformity is not an issue. Modest variations in thickness are also allowable. Finally, because of the multiplexibility of the readout, the focal plane is drastically simplified. Each MKID tile 
requires only a single cryogenic HEMT amplifier and a pair of coaxial cables for the readout; all of the complicated electronics (for demodulation and decimation) are moved to room temperature, where we can take advantage of the rapid advances in microwave electronics for commercial purposes. Furthermore, in our implementation both the multi-slot antennas that are used to photon-couple the MKIDs and the bandpass filters (lumped-element filters comprised of inductors and capacitors) are also lithographed directly on the chip as part of the same fabrication.

With the DemoCam run of May/June 2010, we have demonstrated the functionality of a complete system, with simultaneous multicolor readout of all the pixels. With simultaneous readout of 126 carriers in $\sim 340 \mathrm{MHz}$ of bandwidth, this is the first 3-color readout of an MKID camera. We verified the performance of the optics, antennas, and on-chip bandpass filters, and measured resonator responsivities in agreement with the values predicted by laboratory measurements. Our initial sensitivities, which we can reasonably expect to improve by a factor of a few, are encouragingly low (a few hundred $\mathrm{mJy} \mathrm{s}^{1 / 2}$ at 230 and $290 \mathrm{GHz}, \sim 3$ times larger at 350 $\mathrm{GHz}$ ), and offer the promise of reaching performance approaching the BLIP limit.

\subsection{Acknowledgments}

The MUSIC project is supported by NSF grant AST-0705157 to the University of Colorado, NASA grant NNGC06C71G to Caltech, the Gordon and Betty Moore Foundation, and the JPL Research and Technology Development Fund. As always, the CSO day crew provided terrific support for the observing run. We are grateful to the Xilinx corporation for their generous donation of the FPGAs needed for the readout electronics. Jack Sayers was supported by a NASA Postdoctoral Program Fellowship and James Schlaerth and Nicole Czakon were partially supported by NASA Graduate Student Researchers Program Fellowships.

\subsection{References}

\section{REFERENCES}

[1] Chapman, S.C., Blain, A.W., Smail, I., and Ivison, R.J., "A Redshift Survey of the Submillimeter Galaxy Population", ApJ, 622, 722, (2005).

[2] Czakon, N.G., Day, P.K., Duan, R., Gao, J., Glenn, J., Golwala, S.R., Hollister, M.I., LeDuc, H.G., Maloney, P.R., Mazin, B.A., Nguyen, H.T., Noroozian, O., Sayers, J., Schlaerth, J., Siegel, S., Vaillancourt, J.E., Vayonakis, A., Wilson, P. and Zmuidzinas, J., "Optimization of MKID Noise Performance Via Readout Technique for Astronomical Applications", Proceedings of the SPIE, 7741-26, (2010).

[3] Day, P.K., LeDuc, H.G., Mazin, B.A., Vayonakis, A., and Zmuidzinas, J., "A broadband superconducting detector suitable for use in large arrays", Nature, 425, 817, (2003).

[4] Day, P.K.,Czakon, N.G., Duan, R., Gao, J., Glenn, J., Golwala, S.R., Hollister, M.I., LeDuc, H.G., Maloney, P.R., Mazin, B.A., Nguyen, H.T., Noroozian, O., Sayers, J., Schlaerth, J., Siegel, S., Vaillancourt, J.E., Vayonakis, A., Wilson, P. and Zmuidzinas, J., "A slot array antenna for a millimeter/submillimeter-wave focal plane", Proceedings of the SPIE, 7741-80, (2010).

[5] Duan, R., Czakon, N.G., Day, P.K., Gao, J., Glenn, J., Golwala, S.R., Hollister, M.I., LeDuc, H.G., Maloney, P.R., Mazin, B.A., Nguyen, H.T., Noroozian, O., Sayers, J., Schlaerth, J., Siegel, S., Vaillancourt, J.E., Vayonakis, A., Wilson, P., and Zmuidzinas, J., "An open-source software-defined radio readout for MKIDs", Proceedings of the SPIE, 7741-67, (2010).

[6] Gao, J., Vayonakis, A., Kumar, S., Zmuidzinas, J., Daal, M., Sadoulet, B., Mazin, B., K. Day, P., and Leduc, H.G., "Experimental evidence for a surface distribution of two-level systems in superconducting lithographed microwave resonators", Appl. Phys. Letters, 92, 152505, (2008).

[7] Gao, J., Zmuidzinas, J., Mazin, B.A., LeDuc, H.G., and Day, P.K., "Noise properties of superconducting coplanar waveguide microwave resonators", Appl. Phys. Letters, 90, 102507, (2008).

[8] Gao, J., Daal, M., Martinis, J.M.,, Mazin, B.A., Day, P.K., Leduc, H.G., Vayonakis, A., Sadoulet, B., and Zmuidzinas, J., "A semiempirical model for two-level system noise in superconducting microresonators" Appl. Phys. Letters, 92, 212504, (2008). 
[9] Hollister, M.I., Czakon, N.G., Day, P.K., Duan, R., Gao, J., Glenn, J., Golwala, S.R., LeDuc, H.G., Maloney, P.R., Mazin, B.A., Nguyen, H.T., Noroozian, O., Sayers, J., Schlaerth, J., Siegel, S., Vaillancourt, J.E., Vayonakis, A., Wilson, P., and Zmuidzinas, J., "The cryomechanical design of MUSIC - A novel imaging instrument for millimeter-wave astrophysics at the Caltech Submillimeter Observatory", Proceedings of the SPIE, 7741-56, (2010).

[10] Kumar, S., Vayonakis, A., LeDuc, H.G., Day, P.K., Golwala, S., and Zmuidzinas, J., "Millimeter-Wave Lumped Element Superconducting Bandpass Filters for Multi-Color Imaging", IEEE Transactions on Applied Superconductivity, 19 (3, Part 1), 924, (2009).

[11] Mazin, B., "Microwave Kinetic Inductance Detectors: The First Decade", AIP Conference Proceeding, 1185, 135, (2009).

[12] Monfardini, A., Swenson, L.J., Bideaud, A., Désert, F. X., Yates, S.J.C., Benoit, A., Baryshev, A.M., Baselmans, J.J.A., Doyle, S., Klein, B., Roesch, M., Tucker, C., Ade, P., Calvo, M., Camus, P., Giordano, C., Güsten, R., Hoffmann, C., Leclercq, S., Mauskopf, P., Schuster, K.F., "NIKA: A millimeter-wave kinetic inductance camera", Astr. Ap., in press, (2010).

[13] Noroozian, O., Gao, J., Zmuidzinas, J., LeDuc, H.G., and Day, P.K., "Two-Level-System noise reduction for Microwave Kinetic Inductance Detectors", AIP Conference Proceeding, 1185, 148, (2009).

[14] Sayers, J., Czakon, N.G., Day, P.K., Duan, R., Gao, J., Glenn, J., Golwala, S.R., Hollister, M.I., LeDuc, H.G., Maloney, P.R., Mazin, B.A., Nguyen, H.T., Noroozian, O., Schlaerth, J., Siegel, S., Vaillancourt, J.E., Vayonakis, A., Wilson, P. and Zmuidzinas, J., "Optics for MUSIC: a New (Sub)millimeter Camera for the Caltech Submillimeter Observatory", Proceedings of the SPIE, 7741-32, (2010).

[15] Sayers, J., Golwala, S.R., Ade, P.A.R., Aguirre, J.E., Bock, J.J., Edgington, S.F., Glenn, J., Goldin, A., Haig, D., Lange, A.E., Laurent, G.T., Mauskopf, P.D., Nguyen, H.T., Rossinot, P., and Schlaerth, J., "Studies of Millimeter-wave Atmospheric Noise above Mauna Kea", ApJ, 708, 1674, (2010).

[16] Schlaerth, J., Czakon, N.G., Day, P.K., Duan, R., Gao, J., Glenn, J., Golwala, S.R., Hollister, M.I., LeDuc, H.G., Maloney, P.R., Mazin, B.A., Nguyen, H.T., Noroozian, O., Sayers, J., Siegel, S., Vaillancourt, J.E., Vayonakis, A., Wilson, P., and Zmuidzinas, J. "MKID multicolor array status and results from DemoCam", Proceedings of the SPIE, 7741-8, (2010).

[17] Schlaerth, J., Vayonakis, A., Day, P.K., Glenn, J., Gao, J., Golwala, S., Kumar, S., Leduc, H.G., Mazin, B.A., Vaillancourt, J., and Zmuidzinas, J., "A Millimeter and Submillimeter Kinetic Inductance Detector Camera", J. Low Temp. Phys., 151, 384, (2008).

[18] Tellado, J., and Cioffi, J.M., "PAR reduction in multicarrier tranmission systems", ANSI document T1E1.4/98-367, Sacramento, CA, Dec. 1997. 